\title{
Excitability of Spinal Neurons During a Short Period of Relaxation Im- agery
}

\author{
Toshiaki Suzuki $^{1,2,}$, Yoshibumi Bunno ${ }^{2}$, Chieko Onigata ${ }^{2}$, Makiko Tani ${ }^{1,2}$ and Sayuri Uragami ${ }^{2}$ \\ ${ }^{I}$ Graduate School of Health Sciences, Graduate School of Kansai University of Health Sciences, Japan \\ ${ }^{2}$ Clinical Physical Therapy Laboratory, Faculty of Health Sciences, Kansai University of Health Sciences, Japan
}

\begin{abstract}
To study spinal neuron function during relaxation imagery, we analyzed F-waves of the left thenar muscles in 15 healthy subjects ( 9 males, 6 females; mean age, $27.9 \pm 9.2$ years) after stimulating the left median nerve at the wrist. Fwaves were recorded in a resting position and during and after a 1-min period of relaxation imagery; they were recorded immediately after and at 5, 10, and 15 min after the relaxation imagery period. Persistence, F/M amplitude ratio, and latency were analyzed from the recorded F-waves.

No significant differences were observed in the abovementioned parameters obtained at rest, during, or after relaxation imagery (at any time points). However, the F/M amplitude ratio, as an index of spinal neuron excitability, was used to separate subjects into those with lower excitability, unchanged excitability, and higher excitability than the resting F/M ratios. In our subjective evaluation of the relaxation imagery methods, almost all subjects in the lower excitability group said they performed "relaxation imagery of the hand and thumb." In the higher excitability, almost all subjects said they performed "relaxation imagery of the whole body."

These results indicate that a 1-min period of relaxation imagery does not change the excitability of spinal neurons in healthy subjects. However performing relaxation imagery focused on decreasing muscle tone in a specific area may decrease the excitability of spinal neurons.
\end{abstract}

Keywords: Relaxation imagery, Spinal neuron, F-wave.

\section{INTRODUCTION}

To decrease the muscle tone in affected arms of patients with cerebrovascular disease, we often perform physical therapy using relaxation imagery, which involves actively relaxing the brain. We experienced cases where relaxation imagery was effective in patients who are unable to control their muscles because of cerebrovascular disease.

In this study, we investigated spinal neuron excitability in healthy subjects by analyzing F-waves before, during, and after a 1-min period of relaxation imagery. This study represents a preliminary investigation for establishing physical therapy techniques using relaxation imagery. In addition, we investigated the relationship between spinal neuron excitability during relaxation imagery and the method of relaxation imagery as subjectively described by the study participants.

\section{MATERIALS AND METHODOLOGY}

\subsection{Subjects}

In total, 15 healthy volunteers ( 9 males, 6 females; mean age, 27.9 years) participated in the study. Written informed

*Address correspondence to this author at the Graduate School of Health Sciences, Graduate School of Kansai University of Health Sciences, 2-11-1, Wakaba, Kumatori, Sennan, Osaka 590-0482, Japan;

Tel: +81-72-453-8374; Fax: +81-72-453-8798; E-mail: suzuki@kansai.ac.jp consent was obtained from all subjects. This study was approved by the Research Ethics Committee at Kansai University of Health Sciences. The experiments were conducted in accordance with the Declaration of Helsinki. The authors of this study report no conflicts of interest.

\subsection{F-wave During Motor Imagery}

Subjects were maintained in a comfortable supine position with external rotation of both shoulder joints. The skin was prepared with an abrasive gel to maintain the impedance below $5 \mathrm{~K} \Omega$. A Viking Quest electromyography machine (Natus Medical Inc., CA, USA) was used to record F-waves. We measured F-waves of the left thenar muscles using a pair of round disks attached to the skin with collodion and placed over the belly and on the bone of the metacarpal phalangeal joint of the thumb. Measurements were taken after stimulating the left median nerve at the wrist, both at rest and under relaxation imagery condition. The stimulating electrodes comprised a cathode placed over the left median nerve $3 \mathrm{~cm}$ proximal to the palmar crease of the wrist joint and an anode placed $2 \mathrm{~cm}$ further proximally. The maximal stimulus was determined by delivering $0.2 \mathrm{~ms}$ square-wave pulses of increasing intensity to elicit the generation of a maximal muscle wave (M-wave). Stimuli of supramaximal intensity (adjusted up to $20 \%$ higher than the maximal stimulus to record the maximal M-wave generated) were delivered to the median nerve at $0.5 \mathrm{~Hz}$ for acquisition of F-waves. The bandwidth filter was $2-3 \mathrm{kHz}$. 


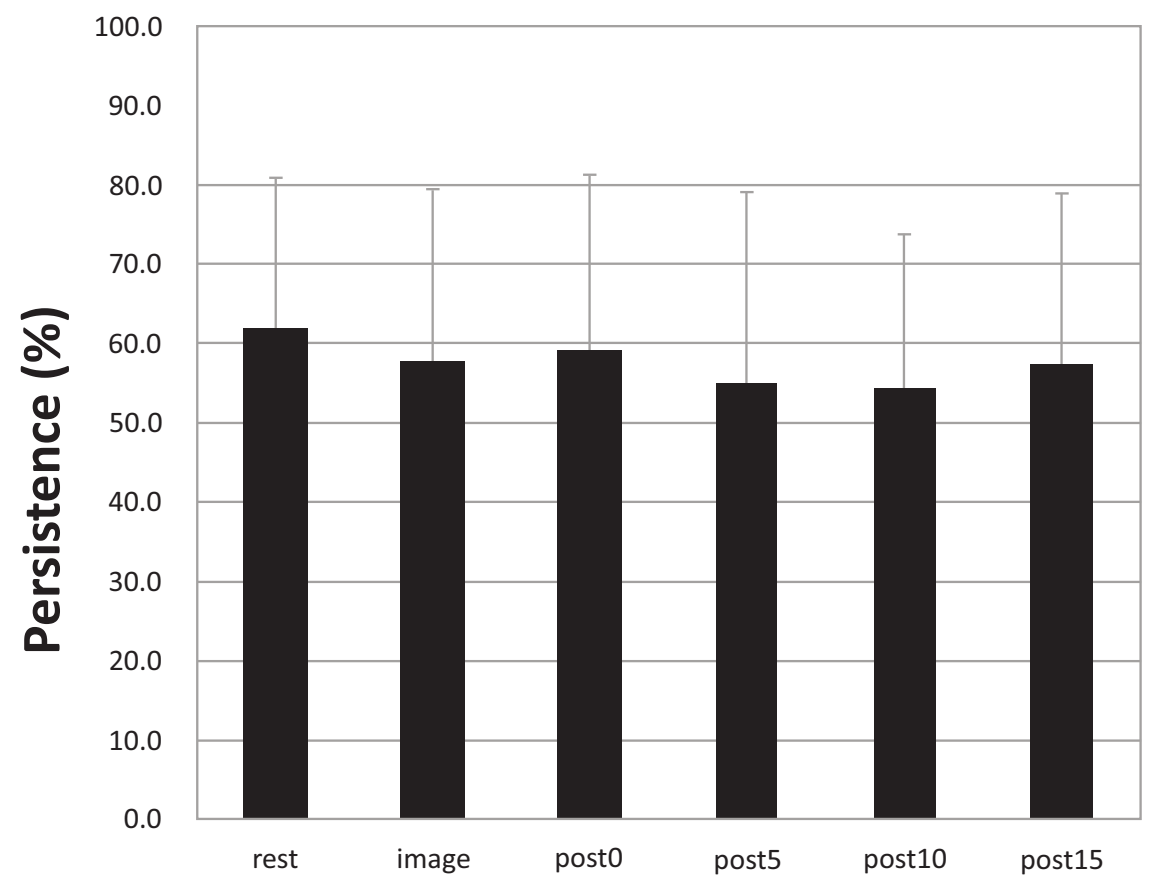

rest:resting position, image: during relaxation imagery, post0: immediately after imagery, post5: 5 min after imagery, post10:10min after imagery, and at 5, 10, and 15 min after imagery

Fig. (1). Persistence in the resting position, during the 1-min relaxation imagery period, immediately after imagery, and at 5, 10, and 15 min after imagery.

In the resting condition, we measured the F-wave during relaxation. For the relaxation imagery condition, F-waves were recorded while the subjects were in a resting position and performing relaxation imagery for $1 \mathrm{~min}$, which was performed immediately after and at 5, 10, and $15 \mathrm{~min}$ after the 1-min relaxation imagery period.

For the subjective evaluation after the test, we asked the subjects "Where and how did you image during the relaxation imagery".

\subsection{Data Analysis}

F-waves from 30 trials were analyzed with respect to persistence, F/M amplitude ratio, and latency. Persistence was defined as the number of measurable F-wave responses divided by 30 trials of supramaximal stimulation. The F/M amplitude ratio was defined as the mean amplitude of all responses divided by the amplitude of the M-wave. Latency was defined as the mean latency from the time of stimulation to the onset of a measurable F-wave.

Dunnett's test was used to compare results between the resting condition and the conditions during and after relaxation imagery.

\section{RESULTS}

There were no significant differences between the abovementioned parameters obtained at rest and during and after relaxation imagery at all time points (Figs. 1-3).

However, the $\mathrm{F} / \mathrm{M}$ amplitude ratio, as an index of spinal neuron excitability, was lower in 6 subjects (lower excitabil- ity group), unchanged in 1 subject (unchanged excitability group), and higher in 8 subjects (higher excitability group) during the 1-min relaxation imagery period than at rest (Figs. 4, 5).

The F/M amplitude ratio at $10 \mathrm{~min}$ after the 1-min relaxation imagery was higher than that at rest, although it was not significant.

In our subjective evaluation of the method of relaxation imagery, of the 6 subjects in the lower excitability group, 4 said they performed "relaxation imagery of the hand and thumb" and 2 said they performed "relaxation imagery of the whole body." Of the 8 subjects in the higher excitability group, 7 said they performed "relaxation imagery of the whole body," and 1 said they performed "relaxation imagery of the hand and thumb."

\section{DISCUSSION}

To decrease the tone of hypertonic muscles in patients with cerebrovascular disease, we usually perform techniques such as continued muscle stretching and electric stimulation.

We believe that performing relaxation imagery for decreasing muscle tone may be useful as a new type of physical therapy.

There are few studies that have reported using relaxation imagery. Fujisawa et al. [1] and Taniguchi et al. [2] reported that both the persistence and $\mathrm{F} / \mathrm{M}$ amplitude ratio were decreased during continued resting for 3 hours. In these studies, healthy subjects were asked to perform positive resting for a long time. 


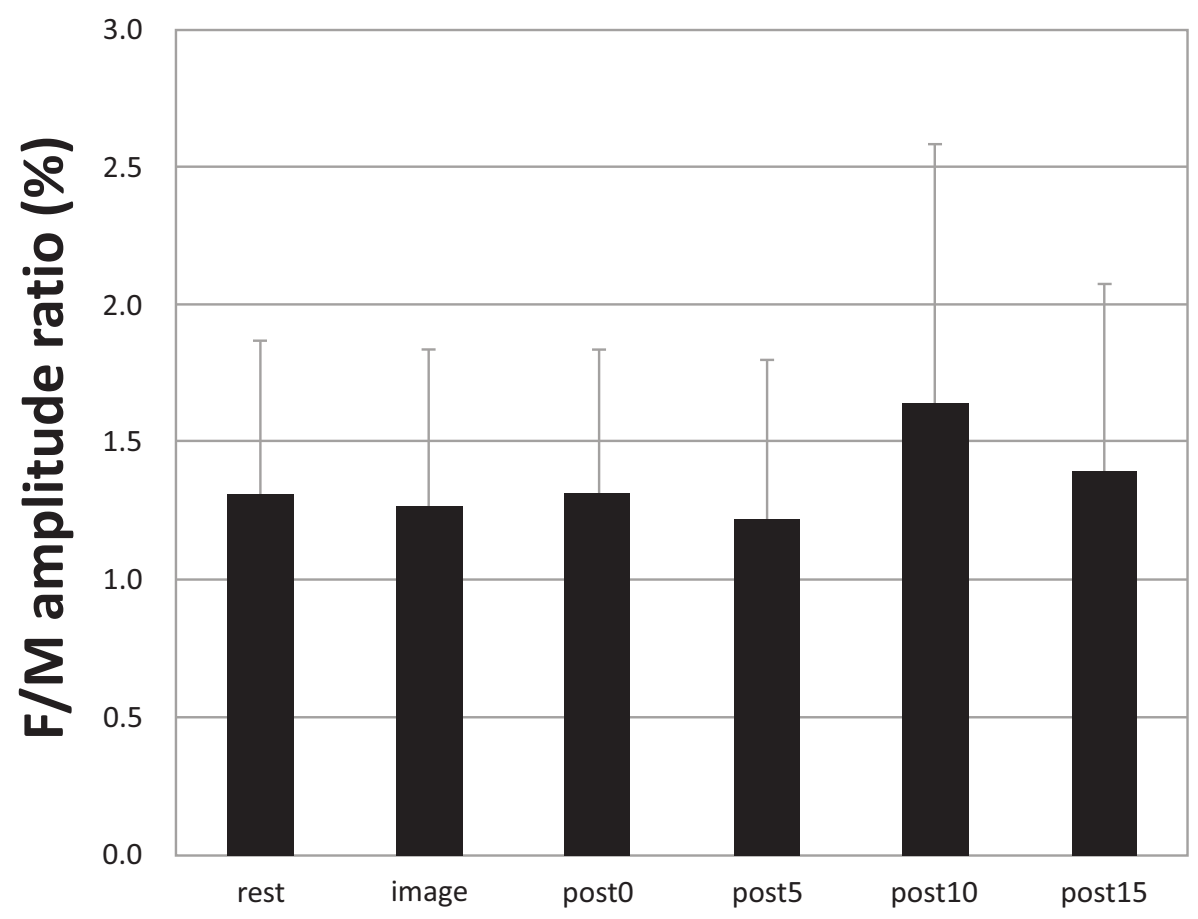

rest:resting position, image: during relaxation imagery, post0: immediately after imagery, post5: 5 min after imagery, post10: 10min after imagery, post15: 15 min after imagery

Fig. (2). F/M amplitude ratio in the resting position, during the 1-min relaxation imagery period, immediately after imagery, and at 5, 10, and 15 min after imagery.

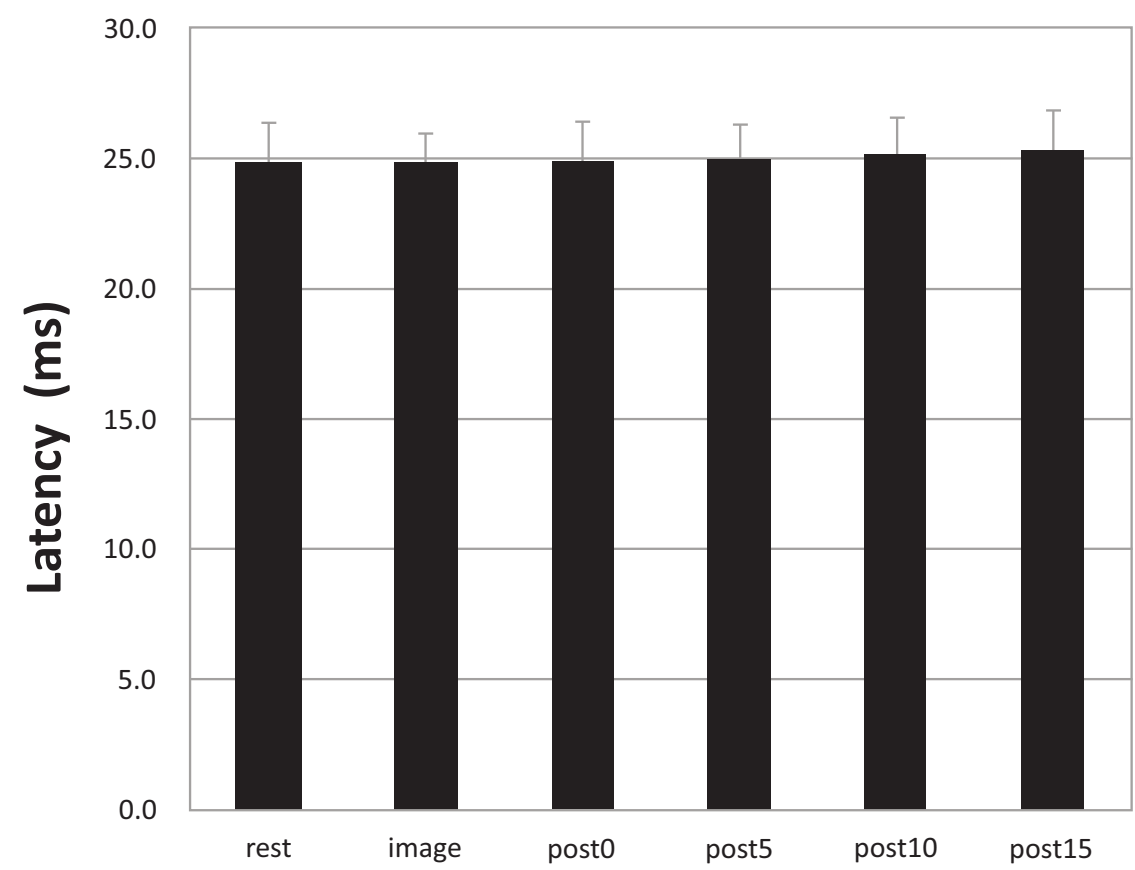

rest:resting position, image: during relaxation imagery, post0: immediately after imagery, post5: 5 min after imagery, post10: 10min after imagery, post15: 15 min after imagery

Fig. (3). Latency in the resting position, during the 1-min relaxation imagery period, immediately after imagery, and at 5, 10, and 15 min after imagery. 


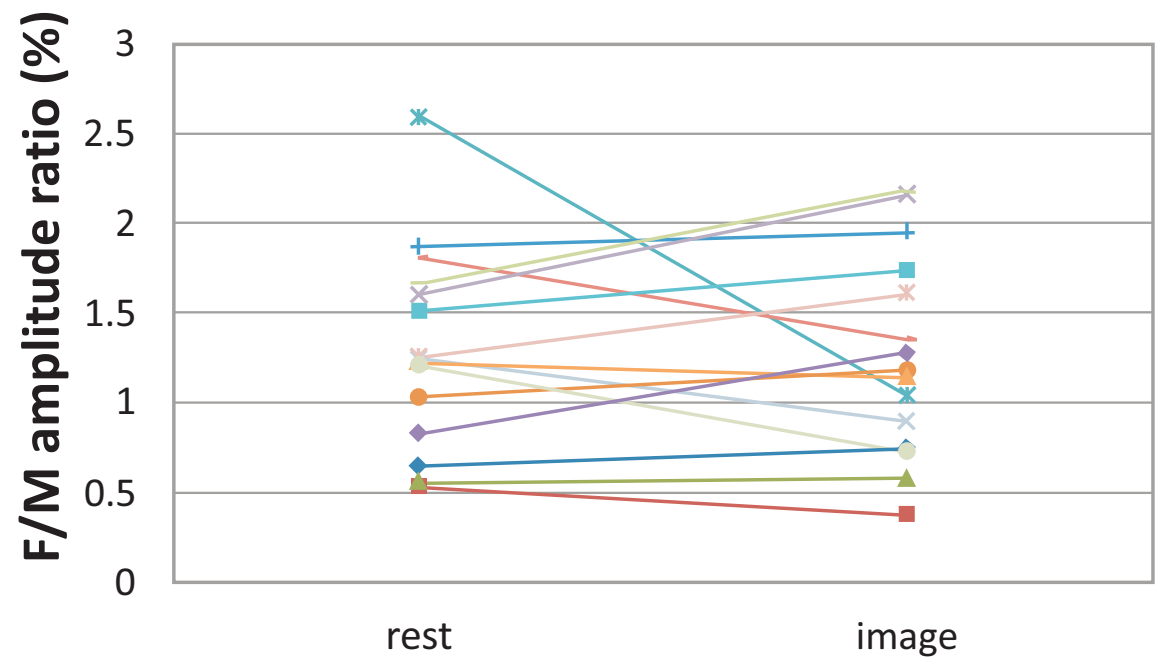

rest: resting position, image: during relaxation imagery

Fig. (4). Change in the F/M amplitude ratio in the resting position and during the 1-min relaxation imagery period.
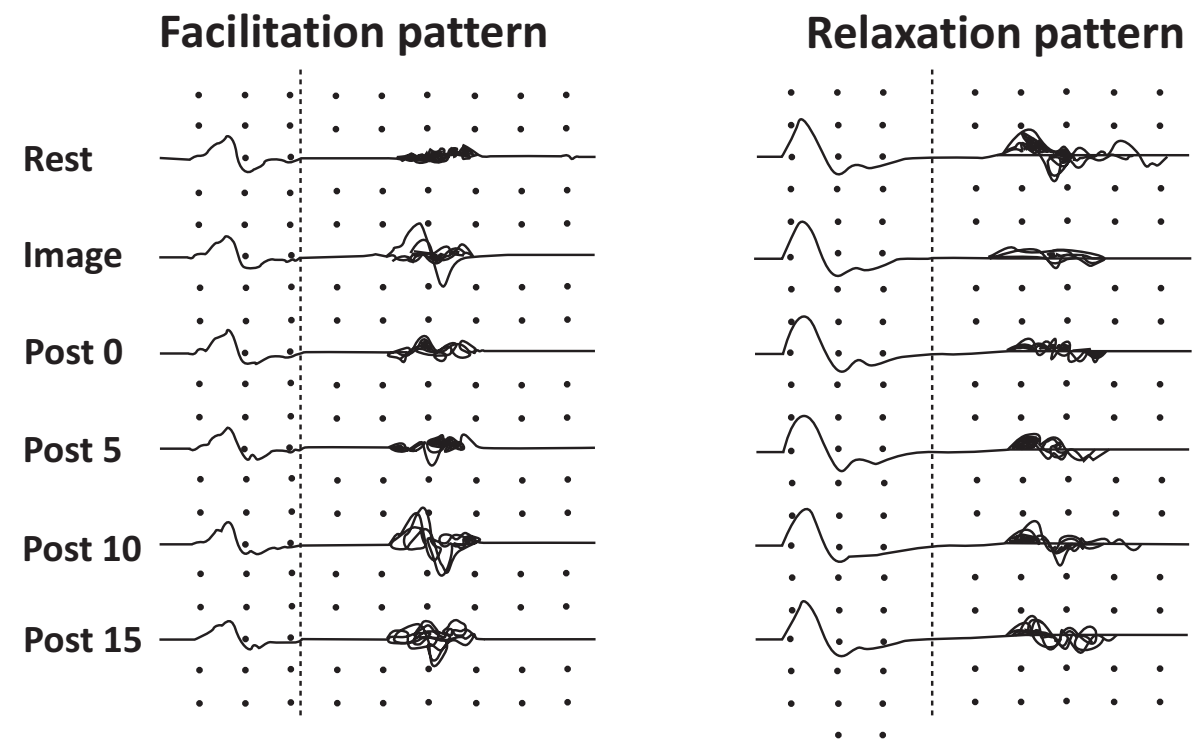

Rest:resting position, image: during relaxation imagery, post0: immediately after imagery, post5: 5 min after imagery, post10:10min after imagery, and at 5, 10, and 15 min after imagery

Fig. (5). Typical Electromyogram patterns for both facilitation and relaxation.

In this study, we investigated spinal neuron excitability in healthy subjects by measuring F-waves at rest and during a 1 -min period of relaxation imagery. This study is a preliminary investigation to establish physical therapy techniques using relaxation imagery. From this study, we found that performing relaxation imagery for 1 min may not decrease spinal neuron excitability. However, the 1-min period of relaxation imagery did decrease spinal neuron excitability in 6 of the 15 subjects. In 4 of these 6 subjects, the reported method of relaxation imagery was "relaxation imagery of the hand and thumb."
The $\mathrm{F} / \mathrm{M}$ amplitude ratio at $10 \mathrm{~min}$ after the 1-min relaxation imagery was higher than that at rest, although it was not significant. Therefore, we hypothesized that this finding indicates the later onset of increased spinal neuron excitability after the rest imagery period.

\section{CONCLUSION}

Based on these results, relaxation imagery for 1 min may not decrease spinal neuron excitability; therefore, we conclude that the optimum relaxation imagery method may be to focus on the body part that requires relaxation. 


\section{CONFLICT OF INTEREST}

The authors declare that there are no conflicts of interest in this study.

\section{ACKNOWLEDGEMENTS}

Declared none.

\section{REFERENCES}

[1] Fujisawa R, Kimura J, Taniguchi S, et al. Effect of volitional relaxation and motor imagery on F wave and MEP: do these tasks affect excitability of the spinal or cortical motor neurons? Clin Neurophysiol 2011; 122: 1405-10.

[2] Taniguchi S, Kimura J, Yamada T, et al. Effect of motion imagery to counter rest-induced suppression of F-wave as a measure of anterior horn cell excitability. Clin Neurophysiol 2008; 119: 1346-52.

Received: November 26, 2013

Revised: December 16, 2013

Accepted: December 18,2013

(C) Suzuki et al.; Licensee Bentham Open.

This is an open access article licensed under the terms of the Creative Commons Attribution Non-Commercial License (http://creativecommons.org/licenses/by-nc/3.0/) which permits unrestricted, non-commercial use, distribution and reproduction in any medium, provided the work is properly cited. 